${ }^{1}$ Corporación contra el cáncer de mama Yo Mujer.

${ }^{2}$ Centro de Referencia en Salud Cordillera Oriente, Santiago, Chile. aPsicóloga, Psicooncóloga. bPsicóloga.

Este trabajo no tuvo apoyo financiero. Basado en la conferencia "¿Contra qué se lucha cuando se lucha?", dictada por Daniela Rojas durante el Simposio Latinoamericano de Gastroenterología Oncológica (SLAGO) año 2013, en Viña del Mar, Chile. Conflictos de intereses: ninguno que declarar.

Recibido el 23 de septiembre de 2014, aceptado el 30 de diciembre de 2014.

Correspondencia a: Loreto Fernández Ricardo Lyon 249, depto. 31, Santiago, Chile. loretofernandez2@gmail.com

\section{¿Contra qué se lucha cuando se lucha? Implicancias clínicas de la metáfora bélica en oncología}

\author{
DANIELA ROJAS MIRANDA ${ }^{1, a}$, LORETO FERNÁNDEZ GONZÁLEZ ${ }^{1,2, b}$
}

\section{Clinical implications of the "war against cancer"}

This article discusses the origin and implications of the "war on cancer" metaphor. Commonly present in mass media, the "war on cancer" notion circulates also among patients, their loved ones, their support networks, and oncological multidisciplinary teams. In our view when cancer is uprooted of its illness status, and conceptualized as an "enemy", myths about disease and those who suffer it (especially the idea of psychogenesis) are strengthened. Two topics in which the war metaphor is particularly problematic in the clinical context, are analyzed in depth. The first one is the relationship between the oncologic patient and his or her loved ones and support networks. When patients are insistently prompted to fight the disease and think positive, the expression of emotions associated to the adaptive process of receiving a diagnosis of cancer may be inhibited. Secondly, the war metaphor promotes an authoritarian view among the health teams and on the physician-patient relationship, undermining the patent's autonomy in the decision-making process, which may affect his global quality of life. Also, it encourages emotional isolation, concealment of psychiatric symptoms and conspiracies of silence. It is concluded that public policies to avoid the "war on" notion are required. Instead, education of the general population about wrong beliefs about cancer should be encouraged.

(Rev Med Chile 2015; 143: 352-357)

Key words: Adaptation, psychological; Attitude to Health; Metaphor; Neoplasms.

\section{A} lo largo de cada año, son numerosas las fechas que abordan la temática del cáncer a través de diversos medios e iniciativas. Ejemplos de esto son el Día Mundial contra el Cáncer (4 de febrero) apoyado por la $\mathrm{OMS}^{1}$ y en octubre, el Mes Internacional de Sensibilización sobre el Cáncer de mama. Durante ese mes, cada año es más frecuente ver una gama de activaciones tanto del sector público como privado, consistente en artículos, reportajes y campañas de prevención y detección precoz de esta patología ${ }^{2,3}$. La mayoría van orientadas a poner el tema sobre la mesa a través de testimonios e historias personales ${ }^{4}$, motivar el autoexamen mamario (aunque su ineficacia como herramienta de detección precoz es debatida hace más de una década $)^{5,6}$ o educar sobre la importancia de la mamografía anual después de los 40 años. Más allá de la estrategia utilizada, o del sector de donde provenga la iniciativa, suele unirlas un mismo motivo: una "invitación" a hacerse parte de "la lucha contra el cáncer".

El abordaje del cáncer con un lenguaje bélico, no es nuevo, está ampliamente utilizado internacionalmente y parece un recurso válido cada vez que hay que referirse a la enfermedad. Es frecuente ver en televisión o en revistas, reportajes sobre la "lucha" de los pacientes oncológicos, así como enterarnos del fallecimiento de un personaje público 
Implicancias clínicas de la "Lucha contra el cáncer" - D. Rojas Miranda et al

con titulares que plantean que tal persona "perdió" o "no pudo ganar la batalla contra el cáncer".

El presente artículo busca reflexionar acerca del origen y las implicancias del uso de estas retóricas en torno al cáncer. Más allá de su utilización en medios de comunicación, la metáfora bélica permea también los equipos médicos y circula a diario indistintamente entre tratantes, pacientes y sus seres queridos. La noción de la "lucha contra el cáncer” se presenta como un discurso transversal, independiente del rol y el nivel de conocimiento técnico que se tenga sobre la enfermedad. Pero, ¿ de dónde viene esta concepción, qué efectos tiene y cómo dialoga con otras creencias sobre el cáncer?

A nuestro juicio, el cáncer, desarraigado de su estatus de enfermedad y conceptualizado como un enemigo con conciencia y voluntad (se suele escuchar que el cáncer "se aprovecha de", "se alimenta de" o "elige a"), acrecienta los miedos en la población sana, y fortalece los mitos que se tejen en torno a esta patología y a quienes la padecen. En particular, nos interesa reflexionar cómo la noción de la "lucha", promueve una actitud en los pacientes, sus seres queridos, y en los equipos tratantes, que muchas veces dista de ser terapéutica, coaccionando a los pacientes a asumir un discurso y una postura frente a su enfermedad que entorpece los procesos adaptativos y los posibles duelos ligados al curso de la patología oncológica y sus tratamientos, pudiendo tener un impacto negativo en la calidad de vida y salud de los pacientes, tanto física como psicológica.

Quisiéramos profundizar en dos ejes en los cuales nos parece particularmente problemática la utilización de la metáfora bélica y sus consecuencias prácticas en el contexto clínico: 1) El paciente oncológico y sus seres queridos, y 2) La relación médico-paciente.

\section{El origen de la metáfora bélica en torno al cáncer}

La atribución de metáforas a las enfermedades, y en particular al cáncer, no es un fenómeno nuevo ni extraño. Forma parte de las atribuciones antropológicas y estéticas que el ser humano otorga a su experiencia ${ }^{7}$. La misma etimología de la palabra "cáncer", cuyo significado es "cangrejo" en griego antiguo, alude a la similitud de ciertos tumores con este crustáceo ${ }^{8}$.

Respecto de la metáfora bélica, objeto de este artículo, existe poco conocimiento sobre su origen. Podríamos plantear la hipótesis que es una construcción desde la oncología o desde los inicios de la psicooncología, como una forma de concientizar a la población o apoyar a los pacientes oncológicos y sus familias. Si bien hay antecedentes de que la noción de "luchar" contra el cáncer había sido utilizada ya en las primeras décadas del siglo $\mathrm{XX}^{9}$, su instauración definitiva se sitúa lejos del mundo de la medicina, la psicología o los mismos pacientes. La "lucha", o "guerra contra..." (según la traducción utilizada del inglés "war on...”) comenzó a difundirse en 1971, postguerra de Vietnam, cuando el presidente de los Estados Unidos de Norteamérica (EEUU), Richard Nixon, firmó el Acta Nacional del Cáncer (si bien en el texto no hay una alusión directa al término bélico $)^{10}$. A través de esta iniciativa, reconocida consensuadamente como el inicio de la "Guerra contra el Cáncer” contemporánea, impulsó económica y políticamente la investigación con el fin de "conquistar esta terrible enfermedad"11 (traducción y cursivas son nuestras) en el corto plazo. Nixon, que solía militarizar las políticas sociales que ponía en marcha, logró instalar a través de esta estrategia político-comunicacional, una particular forma de enfrentar la patología.

La "Guerra contra el Cáncer" cayó en un terreno fértil, desde un gobierno que necesitaba reforzar la noción de supremacía de los EEUU en la ciudadanía (Nixon prometía mediante la iniciativa ser, no sólo la nación más rica, sino también la más sana). De esta forma, el estado robusteció su imagen de control y poder, eligiendo apelar a la vulnerabilidad que despierta una enfermedad percibida como "incontrolable" y devastadora ${ }^{7,12}$.

Hoy, más de 40 años después, países que no tienen la historia bélica ni conflictos recientes como EEUU y Europa asumen como propio un discurso cargado de estas metáforas, discurso que atraviesa programas de salud y campañas preventivas de detección precoz, tanto estatales como privadas. La industria farmacéutica, las instituciones oncológicas y las iniciativas públicas concernientes a la concientización sobre el cáncer y sus efectos, construyen sus eslóganes en torno al imaginario de la guerra y la lucha, en todo el mundo. Y de este modo, la metáfora bélica se perpetúa y retroalimenta, de forma profusa y palpable, también en los profesionales de la salud $\mathrm{y}$ en los mismos pacientes. 
Así, la idea de que frente a la (temida) enfermedad oncológica se debe adoptar una actitud de lucha, tiñe de exigencias a las personas en tratamiento por cáncer, y a quienes prescriben y facilitan estos tratamientos, sin que necesariamente se identifiquen con este discurso. Surge entonces la pregunta acerca de la funcionalidad de esta metáfora, ya no sólo en el contexto de las campañas masivas, sino en el ámbito tanto clínico como privado del paciente con cáncer y quienes lo rodean.

\section{La "lucha" en el paciente oncológico y sus seres queridos/redes de apoyo}

A pesar de existir protocolos de atención y tratamientos de alcance internacional y nacional, cada paciente oncológico presenta una realidad única e irrepetible, tanto en el curso de su enfermedad, como en su contexto psicosocial. No obstante, ciertos patrones emergen a repetición. Uno de estos patrones es la presencia de la metáfora bélica. Es frecuente escuchar a los pacientes comentar ( $\mathrm{y}$ a veces lamentarse) cómo su entorno ha desarrollado una actitud donde se le insta a "no rendirse ante la enfermedad" y a "dar la pelea/batalla/lucha contra el cáncer". En la cotidianeidad esto se traduce en verdaderas exigencias al paciente, tales como "hacer una vida normal", "no pensar cosas negativas", "no llorar", etc. Muchos de estos mensajes transmiten un discurso que destaca y fomenta la disposición a "pensar positivo" para, de este modo, "no deprimirse", cuestión que supuestamente comprometería el pronóstico oncológico. No debemos olvidar que, la mayoría de las veces, estas frases son muestras de apoyo, con la intención de contener, tranquilizar y acompañar al ser querido. Lo interesante es que la creencia a la base de estos mensajes suele ser la psicogénesis del cáncer y el rol de la psique en las probabilidades de sobrevida.

Desde el punto de vista científico, la hipótesis del cáncer como una enfermedad en la que factores psicológicos operarían como "gatillantes" o predisponentes (en mayor o menor medida), ha sido desestimada por múltiples estudios e investigaciones ${ }^{13,14}$. Sin embargo, la interpretación lineal de algunos hallazgos en psiconeuroinmunología (desde la cual se entiende que la tristeza puede generar una baja en el sistema inmune y esto provocar una progresión de la enfermedad, que podría llevar a la muerte prematura), en conjunto con desarrollos teóricos provenientes de diversos campos, ha producido la multiplicación de teorías y perspectivas que refuerzan la idea de la psicogénesis $^{15,16}$. Más allá de la validez científica y de la epistemología subyacente a estas corrientes teóricas, su divulgación en la población general ha provocado no sólo la desconfianza en los tratamientos estándares para el cáncer (poniendo en riesgo la adherencia a los tratamientos o promoviendo la proliferación de terapias alternativas en desmedro de los anteriores). Refuerza, además, en la fantasías de la enfermedad (tanto en población sana como pacientes oncológicos), la idea de que en el origen de la patología es posible encontrar eventos traumáticos desencadenantes (por ejemplo, duelos), o psicopatología predisponente, creencia que genera culpa y angustia en muchos pacientes. Por otro lado, al otorgarle un estatuto de verdad a la noción de que las vivencias, emociones y pensamientos asociados jugarían un papel central en el origen del cáncer, se atribuye una suerte de voluntariedad del sujeto enfermo en el curso y pronóstico de su patología ${ }^{17}$. Frente a esta realidad, una "actitud luchadora", y la metáfora bélica en general, cobra una potencia inusitada.

En efecto, la confusión entre "comunión" mente-cuerpo con "supremacía" de la mente sobre el cuerpo, ha reforzado la idea que una actitud correcta (en este caso la de "lucha"), a través de la represión de pensamientos fatalistas $y$ un manejo emocional eficaz, puede revertir un diagnóstico o un mal pronóstico. Es notable que a pesar de lo arraigada que está dicha creencia en la población y en algunas corrientes psicológicas en boga como, por ejemplo, la Psicología Positiva, tiene un sustento empírico cuyas evidencias son notoriamente cuestionables ${ }^{18}$. Es comprensible que este tipo de ideologías se refuercen a través de metáforas como la bélica. Resulta tentador (y en cierto modo, lógico) pensar que si aquello que provocó el cáncer está en (una disfuncionalidad de) la personalidad o en la historia vital propia, la "lucha" no sólo hace remitir el cáncer, sino que también redime la causa subyacente, es decir, lo "cancerígeno" en primera instancia.

Por otra parte, la insistencia del entorno a que el paciente "luche", "piense positivo" y "no decaiga", sobre exige a quien vive la enfermedad e interfiere en la expresión de emociones propias del proceso de adaptación a los cambios que implica un diagnóstico oncológico y sus tratamientos ad- 
yuvantes. La connotación negativa y patologizante de emociones como la tristeza, la rabia y el miedo contribuye a la estigmatización del paciente con cáncer y a homogeneizar lo que sería una reacción adaptativa "saludable". El mensaje tiene implícita (y a veces explícita) la idea de que la cura de la enfermedad depende de la actitud y estilo de afrontamiento del paciente, otorgando una ilusión de control y un mensaje no realista de lo que puede hacer que el cáncer remita o progrese ${ }^{19}$.

\section{La "lucha" en la relación médico-paciente}

$\mathrm{Al}$ igual que en el apartado anterior, creemos que no hay que desconocer que hay tantas realidades clínicas como pacientes y sus entornos. Sin embargo, es importante visibilizar ciertos fenómenos y condiciones que pueden producirse al interior de los equipos de salud del ámbito oncológico en la relación con los pacientes y sus familias, y qué lugar tiene allí la noción de la lucha contra el cáncer.

Susan Sontag ${ }^{7}$ problematizó hace ya décadas, y desde la posición de ser ella misma una enferma de cáncer, las consecuencias que puede tener para un paciente el pensar el proceso de curación como una guerra. En efecto, la utilización de la metáfora tiene consecuencias concretas, y allí radica la necesidad de visibilizarla. Uno de los resultados lógicos será conceptualizar el propio cuerpo como el campo de batalla (toda guerra se libra en un tiempo y espacio determinados). De esta forma el paciente se vuelve a la vez un enemigo para sí mismo, en tanto es "su" cuerpo el objetivo a atacar.

Lo anterior se vuelve conflictivo desde el punto de la toma de decisiones, tanto del equipo como del paciente. ¿En qué posición queda un paciente cuyo deber ante la enfermedad es luchar una guerra contra su cáncer? Para Mongoven ${ }^{12}$, uno de los principales riesgos de dar un carácter literal a la metáfora bélica, está en la toma de decisiones del paciente, desde el punto de vista de la jerarquía militar que implica este abordaje. El paciente pierde autonomía, ya que, al ser el campo de batalla, se transforma en el objetivo enemigo contra el que se lucha y, además, un sujeto con menor jerarquía que debe acatar decisiones médicas sin cuestionar, tal como un soldado no puede desobedecer órdenes superiores. En este proceso el paciente queda alienado de su propio cuerpo, al haber especialistas que se sitúan en el campo médico desde la perspectiva de la táctica militar, quedando a disposición de este, favoreciendo una visión autoritaria en los equipos y en la relación médico-paciente. Además, puede potenciar la estigmatización de algunos pacientes que tiendan a cuestionar los actos y decisiones médicas ${ }^{12}$, etiquetándolos de poco dóciles, y de ser "pacientes de difícil manejo", o bien psicopatologizando su conducta ("paciente oposicionista", "paciente negador"). Si bien la "negación" como fenómeno psíquico y mecanismo de afrontamiento, ha sido descrita tanto en los tratantes como en los pacientes $^{20}$, pareciera pasarse por alto que en el caso del cáncer esto reside en cómo la enfermedad es conceptualizada en su totalidad, tanto a nivel social, interpersonal e individual.

Esto también puede volverse en contra del paciente, desde el punto de vista de la optimización de los cuidados y la calidad de vida del mismo sujeto. Un ejemplo frecuente es el temor de los pacientes a ser derivados a Cuidados Paliativos, donde la idea de "haber perdido la batalla" es inminente, despertando fantasías de fracaso (rendirse) y desahucio (entregarse al enemigo), no sólo en los pacientes y sus familias sino también en los equipos. Esto produce muchas veces un desmedro en la calidad de vida de los pacientes $y$, paradójicamente, menor sobrevida, en tanto se ha visto que tratamientos más agresivos (o que aún se encuentran en fases experimentales) no necesariamente tienen como corolario mejores tasas de sobrevida ${ }^{21}$.

Además, favorece la postergación de necesidades corporales y la minimización de los efectos adversos de los tratamientos adyuvantes, por mantener la posición de "lucha" (obligarse a levantarse, comer, ocultar la fatiga, náuseas, dolor, etc.). Este enfrentamiento tiende a generar aislamiento en los pacientes y favorecer conspiraciones de silencio al interior de grupos familiares y redes de apoyo. Al silenciar sus emociones, pensamientos recurrentes y síntomas (tanto físicos como psicopatológicos), para no frustrar las expectativas de sus seres queridos y su equipo tratante, se produce una vivencia más solitaria del proceso y, a la larga, mayor sufrimiento, dolor y psicopatología que puede requerir intervenciones tanto psicosociales como farmacológicas ${ }^{22}$. En ese sentido, puede conducir a complicaciones médicas y problemáticas psicosociales de diversa complejidad y manejo. 


\section{Conclusiones}

Hemos analizado el efecto de la metáfora bélica en los pacientes oncológicos, en relación con sus seres queridos y los equipos tratantes. Nos parece central enfatizar las consecuencias negativas que ésta puede tener en la calidad de vida global del paciente y quienes lo rodean, al interferir en los procesos de adaptación tanto físicos como psicosociales que implica el recibir un diagnóstico de cáncer y la consecución de los tratamientos asociados. Además, es importante identificar aquellos sesgos que pueden emerger en la toma de decisiones que involucran a los pacientes, a los cuidadores y los equipos tratantes. En efecto, es necesario visibilizar que la metáfora bélica produce una ideologización particular de la enfermedad al atribuir significados, asignar roles, e introducir valoraciones morales respecto de conductas deseables e indeseables, tanto a la patología misma como a los actores involucrados en ella ${ }^{23}$.

Esta perspectiva resulta injusta para aquellos pacientes y sus familias, cuando la enfermedad no remite. La mayoría de los pacientes y sus tratantes, en los que el cáncer progresa, hicieron todo lo que estuvo a su alcance para superar la enfermedad. Entonces, ¿son perdedores? ¿Sería justo pensar que un paciente que fallece "perdió la batalla", "no luchó lo suficiente", "tenía conflictos o duelos no resueltos" o "no tenía suficiente apego a la vida"?

Más importante aun: es esencial introducir la distinción de que no toda pérdida implica una derrota para los actores involucrados. Todo aquel que haya vivido la experiencia del fallecimiento de un paciente con cáncer, ya sea desde un rol profesional o personal, ha podido ser testigo de que quienes han fallecido por esta causa están lejos de ser perdedores de la lucha contra el cáncer.

Identificar el cáncer como un enemigo hacia el cual corresponde desarrollar una actitud de lucha, demoniza aun más la enfermedad, acrecentando en la población las ideas de muerte inminente, tratamientos invasivos, dolor incontrolable, etc. El miedo a la enfermedad no siempre tiene como consecuencia la movilización y adquisición de hábitos de vida saludables o de conductas de autocuidado. Por el contrario, las campañas centradas en educar y transmitir una percepción de control y eficacia sobre la patología, son las que generan mayor cambio de hábitos y realización de exámenes de pesquisa ${ }^{24,25}$.
Educar a la población en cuanto a los mitos y creencias erróneas sobre la enfermedad oncológica, tales como la psicogénesis, constituye una tarea prioritaria tanto para quienes trabajamos en este ámbito, como para quienes diseñan las políticas destinadas a prevenir el cáncer y fomentar su detección precoz. De este modo, la enfermedad puede ser desmitificada y abordarse de una forma más realista, lo que a su vez puede tener un impacto positivo en la costo-efectividad de las intervenciones integrales en torno a la enfermedad oncológica.

\section{Referencias}

1. Organización Mundial de la Salud [Internet]. [Consultado el 3 de mayo de 2014] Día Mundial contra el Cáncer 2014. Disponible en: http://www.who.int/cancer/es/

2. Avon [Internet]. [Consultado el 3 de mayo de 2014] Cruzada contra el cáncer de mama. Disponible en: http://www.avon.es/PRSuite/crusade.page\#

3. haztelamamografiahoy.cl [Internet]. Servicio Nacional de la Mujer; c2013 [Consultado el 3 de mayo de 2014]. Disponible en: http://www.haztelamamografiahoy.cl/

4. Organización Mundial de la Salud [Internet]. Cara a cara con las enfermedades crónicas; c2005 [Consultado el 3 de mayo de 2014]. La lucha de María contra el cáncer. Disponible en: http://www.who.int/features/galleries/ chronic_diseases/maria/01_es.html

5. Harris R, Kinsinger LS. Routinely teaching breast selfexamination is dead. What does this mean? J Natl Cancer Inst 2002; 94: 14201.

6. Hackshaw AK, Paul EA. Breast self-examination and death from breast cancer: a meta-analysis. Br J Cancer 2003; 88 (7): 1047-53.

7. Sontag S. La enfermedad y sus metáforas. $1^{\mathrm{a}}$ edición. Buenos Aires: Random House Mondadori; 2012.

8. Wagener DJ. The History of Oncology. $1^{\text {st }}$ ed. Houten: Springer Uitgeverij; 2009.

9. Holland J, Weiss, T. History of Psycho-Oncology. En: Psycho-oncology. Holland J, Breitbart W, Jacobsen P, Lederberg M, Loscalzo M, McCorckle R, editores. New York: Oxford University Press; 2010. p 3-12.

10. National Cancer Institute [Internet]. Office of Government and Congressional Relations: Legislative History: Public Health Service Act [Consultado el 19 de mayo de 2014]. The National Cancer Act of 1971. Disponible en: http://legislative.cancer.gov/history/phsa/1971.

11. Developmental Therapeutics Program [Internet]. Milestone 1971 [Consultado el 19 de mayo de 2014]. National Cancer Act of 1971. Disponible en: http://dtp.nci.nih. 
gov/timeline/noflash/milestones/M4_Nixon.htm

12. Mongoven A. The war on disease and the war on terror: A dangerous metaphorical nexus? Camb Q of Health Ethic 2006; 15: 403-16.

13. Ranchor A, Sanderman R, Coyne J. Invited commentary: Personality as a causal factor in cancer risk and mortality- time to retire a hypothesis? Am J Epidemiol 2010; 172 (4): 386-8.

14. Johansen C. Psychosocial Factors En: Psycho-oncology. Holland J, Breitbart W, Jacobsen P, Lederberg M, Loscalzo M, McCorckle R, editores. New York: Oxford University Press; 2010. p 57-61.

15. Middleton J. Yo (no) quiero tener cáncer. $1^{\text {a }}$ ed. Santiago: Grijalbo Mondadori; 1996.

16. Teixidor A. Emobiología y medicina [Internet] Barcelona: Assensi Teixidor [Consultado el 23 de julio de 2014] disponible en http://www.assensiteixidor.emobiola.com/ index.php/47-factores-de-la-activacion-del-proceso.

17. Hay L. Usted puede cambiar su vida. 1a ed. Buenos Aires: Urano; 1989.

18. Coyne J, Tennen H. Positive Psychology in Cancer Care: bad science, exaggerated claims, and unproven medicine.
Ann Behav Med 2010; 39: 16-26.

19. Coyne J, Tennen H, Ranchor A. Positive Psychology in Cancer Care: A story line resistant to evidence. Ann Behav Med 2010; 39: 35-42.

20. Denial and decision-making-capacity Arranz P, Barreto P. En: Walsh TD, ed. Palliative Medicine. Philadelphia: Saunders/Elsevier; 2009. p 647-50.

21. Temel J, Greer J, Muzikansky A, Gallagher E, Admane $\mathrm{S}$, Jackson V, et al. Early palliative care for patients with metastatic non-small-cell lung cancer. N Engl J Med 2010; 363: 733-42.

22. Reich M, Mekaoui L. La conspiration du silence en cancérologie: une situation à ne pas négliger. Bull Cancer 2003; 90 (2): 181-4.

23. Haines I. The war on cancer: time for a new terminology. The Lancet 2014; 383: 1883.

24. Witte K, Allen M. A Meta-Analysis of Fear Appeals: Implications for Effective Public Health Campaigns. Health Educ Behav 2000; 27 (5): 591-615.

25. Riquelme $\mathrm{G}$, Concha $\mathrm{X}$, Urrutia MT. Intervenciones educativas para la prevención del cáncer cervicouterino. Rev Chil Obstet Ginecol 2012; 77 (2): 111-5. 